POS PROCEEDINGS

\title{
Chiral restoration of the momentum space quark propagator through Dirac low-mode truncation
}

\author{
Mario Schröck* \\ Institut für Physik, FB Theoretische Physik, Universität Graz, A-8010 Graz, Austria \\ E-mail: mario.schroeck@uni-graz.at
}

\begin{abstract}
We calculate the chirally improved (CI) Landau gauge quark propagator in two flavor lattice Quantum Chromodynamics and study thereon the effects of Dirac low-mode removal. The application of tree-level improvement of the propagator and tree-level correction of the lattice dressing functions removes the leading discretization artifacts. We find the dynamically generated mass in the infrared domain of the mass function to dissolve continuously with the reduction level and furthermore we observe strong suppression of the wave-function renormalization function for small momenta.
\end{abstract}

Xth Quark Confinement and the Hadron Spectrum 8-12 October 2012

TUM Campus Garching, Munich, Germany

\footnotetext{
*Speaker.
} 


\section{Motivation}

The lowest eigenmodes of the Dirac operator are related to the dynamical breaking of the chiral symmetry in QCD via the Banks-Casher relation. In [1,2] we investigated the effects of the removal of the lowest Dirac eigenmodes from the valence quark sector on the hadron mass spectrum in a dynamical lattice QCD simulation. It was found that all hadrons, except for the pions, survive the aforementioned truncation. The masses of hadrons that can be transformed into each other by a chiral rotation became degenerate and surprisingly the value of these masses remained rather large: see for example Fig. 1 where we show the evolution of the vector and axial vector meson masses under Dirac low-mode removal.

The removal of the lowest Dirac eigenmodes, i.e., the removal of the chiral condensate is expected to result in the loss of dynamically generated mass in the valence quarks. Thus, the naive expectation suggests rather light hadrons once the chiral symmetry has been restored. The study [3] aimed at shedding some light on the issue of mass generation in the context of Dirac lowmode removal: here we explored the valence quark propagators that have been used in the previous studies, in a gauge fixed setting in momentum space. Then it is possible to extract at each Dirac low-mode truncation level the mass function of the propagator which manifestly shows the amount of dynamically generated mass in the quarks.

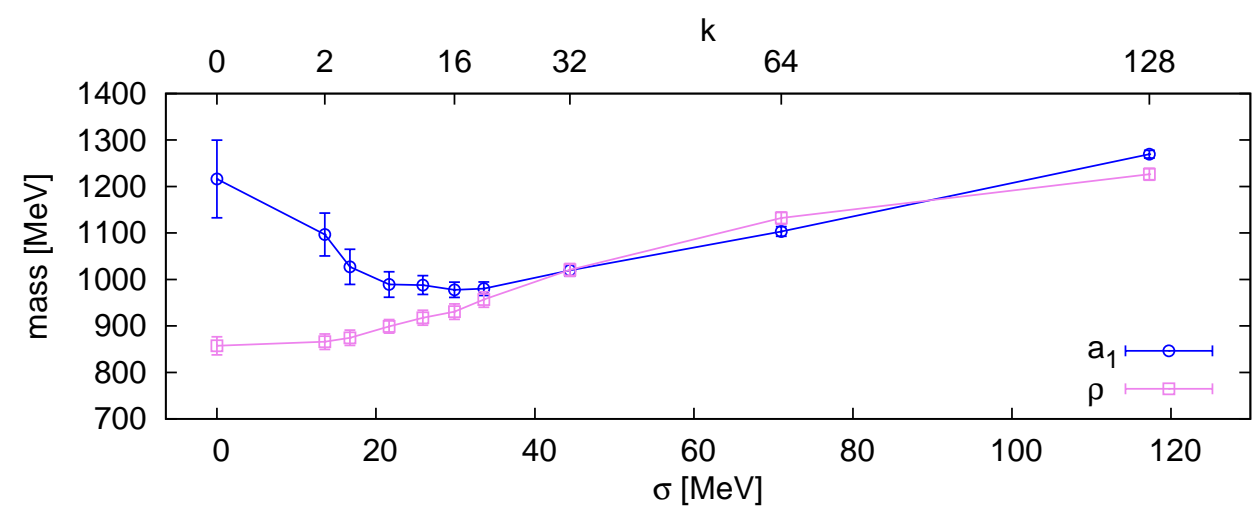

Figure 1: The masses of the vector meson $\rho$ and axial vector meson $a_{1}$ under Dirac low-mode removal: the would be chiral partners become degenerate once the chiral condensate has been removed.

\section{Dirac low-mode truncation}

The lowest eigenmode of the Dirac operator are known to play a crucial role in the dynamical breaking of the chiral symmetry as stated by the Banks-Casher relation. Consequently, when removing the Dirac eigenmodes near the origin, the chiral condensate disappears and chiral symmetry becomes "unbroken" [1].

Consider the Hermitian Dirac operator $D_{5} \equiv \gamma_{5} D$. We denote its (real) eigenvalues with $\mu_{i}$ and the corresponding eigenvectors with $\left|v_{i}\right\rangle$. We write the Dirac operator $D$ in terms of the spectral 
representation of $D_{5}$ :

$$
D=\sum_{i=1}^{N} \mu_{i} \gamma_{5}\left|v_{i}\right\rangle\left\langle v_{i}\right|
$$

Then we can split the quark propagator $S=D^{-1}$ into a low-mode part ( $\mathrm{lm}$ ) and a reduced part (red),

$$
S=\sum_{i \leq k} \mu_{i}^{-1}\left|v_{i}\right\rangle\left\langle v_{i}\left|\gamma_{5}+\sum_{i>k} \mu_{i}^{-1}\right| v_{i}\right\rangle\left\langle v_{i}\right| \gamma_{5} \equiv S_{\operatorname{lm}(k)}+S_{\operatorname{red}(k)}
$$

and hence we obtain the reduced part of the propagator by simply subtracting the low-mode part from the full propagator

$$
S_{\text {red }(k)}=S-S_{\operatorname{lm}(k)} .
$$

It is this reduced part of the propagator which we calculate within a gauge fixed setting in order to extract its momentum space dressing functions.

We use dynamical two flavor gauge field configurations [4, 5] for our study where for the dynamical quarks as well as for the valence quark propagator that we investigate, the so called chirally improved (CI) Dirac operator [6, 7] has been adopted. We gauge fixed the configurations to Landau gauge with the $c u L G T$ code [8] for lattice gauge fixing on graphic processing units (GPUs) with CUDA.

\section{The CI quark propagator from the lattice}

The continuum quark propagator at tree-level reads

$$
S^{(0)}(p)=(i \not p+m)^{-1}
$$

where $m$ denotes the bare quark mass. In manifestly covariant gauges the interacting renormalized quark propagator $S(\mu ; p)$ can be decomposed into a Dirac scalar and a Dirac vector part

$$
S(\mu ; p)=\left(i \not p A\left(\mu ; p^{2}\right)+B\left(\mu ; p^{2}\right)\right)^{-1}
$$

or equivalently

$$
S(\mu ; p)=Z\left(\mu ; p^{2}\right)\left(i \not p+M\left(p^{2}\right)\right)^{-1} .
$$

Here we introduced the wave-function renormalization function $Z\left(\mu ; p^{2}\right)=1 / A\left(\mu ; p^{2}\right)$ and the mass function $M\left(p^{2}\right)=B\left(\mu ; p^{2}\right) / A\left(\mu ; p^{2}\right)$.

The regularized lattice quark propagator $S_{L}(p ; a)$ can be renormalized at the renormalization point $\mu$ with the quark wave-function renormalization constant $Z_{2}(\mu ; a)$,

$$
S_{L}(p ; a)=Z_{2}(\mu ; a) S(\mu ; p) .
$$

The mass function $M\left(p^{2}\right)$ is independent of the renormalization point $\mu$ whereas the wavefunction renormalization function $Z\left(\mu ; p^{2}\right)$ differs at different scales although can be related from different scales by multiplication with a constant.

We extract the functions $M\left(p^{2}\right)$ and $Z\left(p^{2}\right)$ of the CI quark propagator from a the lattice. For details of the extraction see Ref. [9]. 
The lattice quark propagator at tree-level

$$
S_{L}^{(0)}(p)=\left(i a k+a M_{L}^{(0)}(p)\right)^{-1}
$$

differs from the continuum case due to discretization artifacts. The dressing function $A_{L}^{(0)}(p)$ equals one, by construction, and hence $B_{L}^{(0)}(p)$ equals at tree-level the mass function $M_{L}^{(0)}(p)$.

We extract the CI lattice momenta $a k(p)$ from the tree-level propagator and compare it to the analytically derived expression for the $D_{\mathrm{CI}}$ momenta given in [3]. The lattice calculation is consistent with the analytical result, see the r. h. s. plot of Fig. 2.
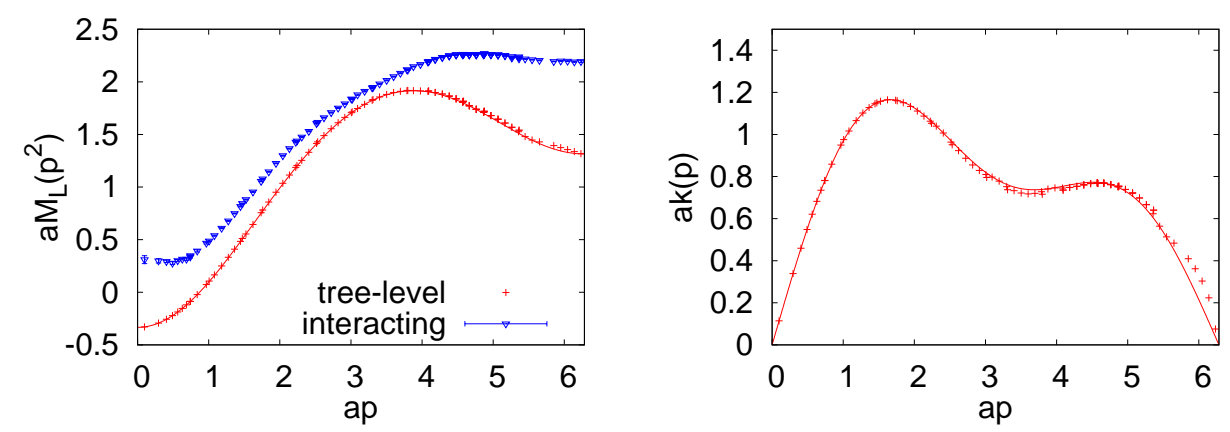

Figure 2: The tree-level mass function (left in red) and lattice CI momenta (right) together with the corresponding analytical curves and the uncorrected interacting mass function (left in blue).

The tree-level mass function $a M_{L}^{(0)}(p)$ which in the continuum is simply the bare mass $m$, is shown in the 1. h. s. plot of the same Fig. 2 (in red), again consistency with the corresponding analytical expression is manifest. As can be seen from the plot, $a M_{L}^{(0)}(p)$ has a zero-crossing with $a M_{L}^{(0)}(0) \approx-0.333$.

When switching from the tree-level case to the non-perturbative full interacting case, the mass function $a M_{L}^{(0)}(p)$ of the 1. h. s. plot of Fig. 2 (red) turns into the interacting lattice mass function $a M_{L}(p)$ (blue).

The shape of $a M_{L}(p)$ is similar to $a M_{L}^{(0)}(p)$ and is clearly altered by discretization errors. To get a handle on the leading lattice artifacts we perform improvement and tree-level correction. For the application of the Symanzik improvement program [10] to reduce the errors of the fermionic action to $\mathscr{O}\left(a^{2}\right)$ we refer to [3] and references therein.

In order to blank out the lattice artifacts which are already present at tree-level, we aim at extracting the deviation of the interacting propagator from its tree-level form. To this end we simply divide the renormalization function $Z_{L}(p)$ by its tree-level form

$$
Z_{L}(p) \rightarrow \frac{Z_{L}(p)}{Z_{L}^{(0)}(p)} .
$$

In order to apply an equivalent tree-level correction to the mass function of the form

$$
a M_{L}(p) \rightarrow \frac{a m M_{L}(p)}{M_{L}^{(0)}(p)}
$$


we have to carry out an additive mass renormalization of the tree-level function $B_{L}^{(0)}(p)$ in order to avoid divergences because of the zero crossing of the tree-level function,

$$
a B_{L}^{(0)}(p) \rightarrow a B_{L}^{(0)}(p)+a m_{\mathrm{add}}
$$

where $a m_{\text {add }}$ is chosen such that $B_{L}^{(0)}(0)=m$, like in the continuum, i.e.

$$
a m_{\mathrm{add}}=a m-a B_{L}^{(0)}(0) \approx 0.344 .
$$

Thus, the multiplicative tree-level correction for the mass function is

$$
a M_{L}(p) \rightarrow \frac{a m M_{L}(p) A_{L}^{(0)}(p)}{B_{L}^{(0)}(p)+m_{\mathrm{add}}} .
$$

\section{Results}

We extract the quark wave-function renormalization function $Z_{L}(p)$ and the quark mass function $M_{L}(p)$ from the reduced propagators of (2.3) while varying the Dirac low-mode reduction level $k$ form 2 to 512. We tree-level improve the modified propagators and apply the aforementioned tree-level correction scheme. The dressing functions from the full propagators together with the dressing functions from the most extreme reduction (512 eigenmodes subtracted) are presented in Fig. 3.
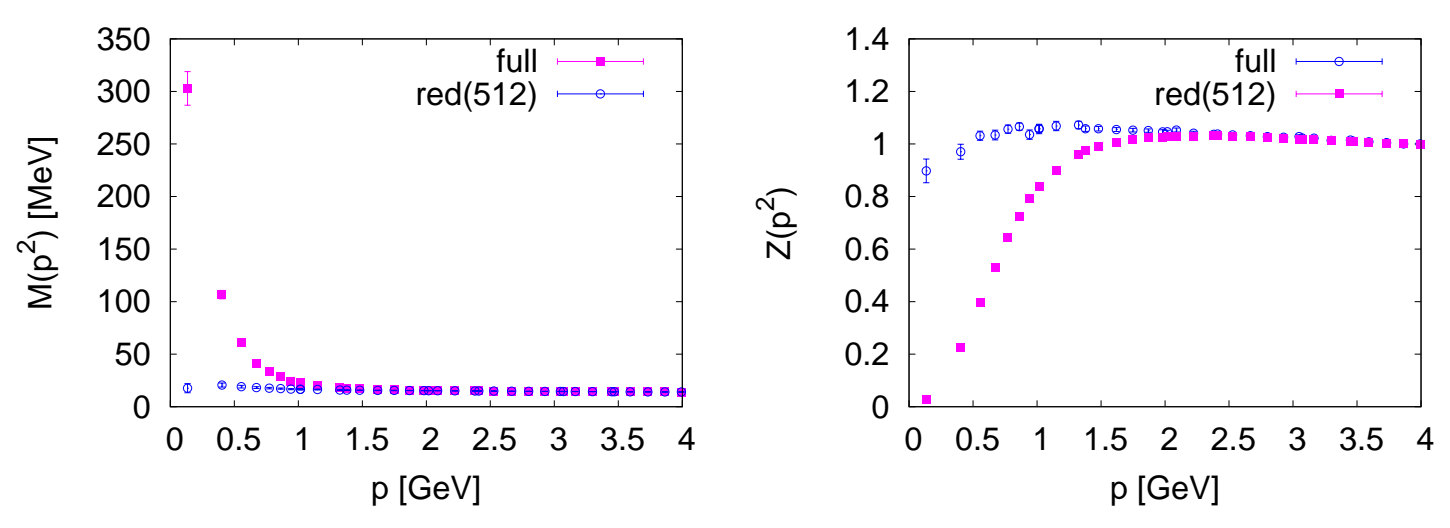

Figure 3: Left: The corrected mass function from the original theory and after having subtracted the lowest 512 Dirac eigenmodes. Right: The same comparison for the wave-function renormalization function of the quark propagator.

The plot reveals amplification of infrared suppression of $Z_{L}(p)$ when subtracting Dirac lowmodes while the range from medium to high momenta becomes not affected at all. Consequently, far traveling quarks are suppressed in this framework.

The mass function $M_{L}(p)$ gets strongly suppressed in the infrared when removing the lowest eigenmodes, the dynamic generation of mass ceases. In Fig. 4 we plot the deep infrared mass of the CI quark propagator from $M_{L}\left(p_{\min }^{2}\right)$, at the smallest available momentum $p_{\min }=0.13 \mathrm{GeV}$, as a function of the Dirac low-mode reduction level. We see that the value of the bare quark mass (horizontal line) is approximately reached at truncation level $k=256$. Note that the reduction level $k$ can be translated to an energy scale which is shown in the lower axis. 


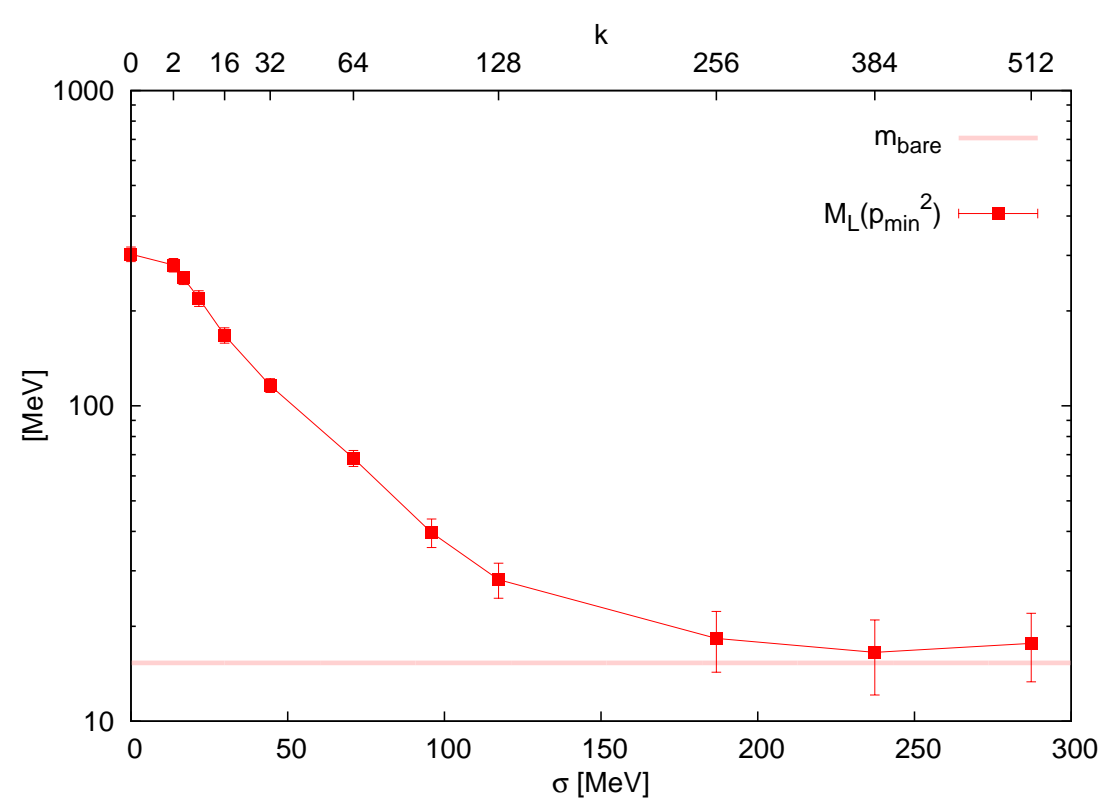

Figure 4: The value of the lattice mass function $M_{L}\left(p^{2}\right)$ at the lowest available momentum $p^{2}$ as a function of the truncation level. The horizontal line is the value of the bare quark mass.

\section{Conclusions}

We found that the dynamically generated mass in the valence quark propagator vanishes after a critical number of the lowest Dirac eigenmodes have been excluded from the simulation. This is in accordance with the Banks-Casher relation which relates the Dirac low-modes to the chiral condensate. Thus, the rather large mass of hadrons which are built out of such truncated quark propagators, see Fig. 1 and References $[1,2]$ cannot be explained by the dynamical breaking of the chiral symmetry. Interestingly, however, we find an intermediate Dirac low-mode truncation regime where the dynamically broken chiral symmetry seems to be restored, as indicated by the degeneracy of the masses of the chiral partner mesons $\rho$ and $a_{1}$ but, at the same time, the quark propagator still shows a significant amount of dynamically generated mass. Similar findings were obtained by the authors of [11] who performed a hadron spectroscopy on gauge field configurations from which center vortices have been cut out.

\section{Acknowledgments}

I thank C. B. Lang and L. Ya. Glozman for helpful discussions. Support by the Research Executive Agency (REA) of the European Union under Grant Agreement PITN-GA-2009-238353 (ITN STRONGnet) is gratefully acknowledged.

\section{References}

[1] C. B. Lang and M. Schröck, Unbreaking chiral symmetry, Phys.Rev. D84 (2011) 087704 , [arXiv: 1107.5195$]$. 
[2] L. Y. Glozman, C. B. Lang, and M. Schröck, Symmetries of hadrons after unbreaking the chiral symmetry, Phys.Rev. D86 (2012) 014507, [arXiv: 1205 . 4887].

[3] M. Schröck, The chirally improved quark propagator and restoration of chiral symmetry, Phys.Lett. B711 (2012) 217-224, [arXiv:1112.5107].

[4] C. Gattringer, C. Hagen, C. B. Lang, M. Limmer, D. Mohler, and A. Schäfer, Hadron Spectroscopy with Dynamical Chirally Improved Fermions, Phys. Rev. D79 (2009) 054501, [arXiv:0812.1681].

[5] BGR [Bern-Graz-Regensburg] Collaboration, G. P. Engel, C. B. Lang, M. Limmer, D. Mohler, and A. Schäfer, Meson and baryon spectrum for QCD with two light dynamical quarks, Phys. Rev. D82 (2010) 034505, [arXiv:1005.1748].

[6] C. Gattringer, A new approach to Ginsparg-Wilson fermions, Phys. Rev. D63 (2001) 114501, [hep-lat/ 0003005$]$.

[7] C. Gattringer, I. Hip, and C. B. Lang, Approximate Ginsparg-Wilson fermions: A first test, Nucl. Phys. 597 (2001) B451, [hep-lat/ 0007042$].$

[8] M. Schröck and H. Vogt, Coulomb, Landau and Maximally Abelian Gauge Fixing in Lattice QCD with Multi-GPUs, arXiv:1212.5221.

[9] J. I. Skullerud and A. G. Williams, The Quark propagator in momentum space, Nucl.Phys.Proc.Suppl. 83 (2000) 209-211, [hep-lat/9909142].

[10] K. Symanzik, Continuum Limit and Improved Action in Lattice Theories. 1. Principles and phi**4 Theory, Nucl.Phys. B226 (1983) 187.

[11] E.-A. O’Malley, W. Kamleh, D. Leinweber, and P. Moran, SU(3) centre vortices underpin confinement and dynamical chiral symmetry breaking, Phys.Rev. D86 (2012) 054503, [arXiv: 1112.2490 ]. 